\title{
Dil and DiO: versatile fluorescent dyes for neuronal labelling and pathway tracing
}

\author{
Marcia G. Honig and Richard I. Hume
}

The fluorescent carbocyanine dyes dil and diO have an extensive history of use in cell biology, but their use as neuronal tracers is relatively recent. We found in 1985 that these molecules were excellent retrograde and anterograde tracers in the developing nervous system. We went on to show that these dyes were retained in neurons placed in culture, that they initially labelled the processes as well as the cell bodies of cultured neurons, and that they were seemingly non-toxic ${ }^{1,2}$. We suggested that the major mechanism of translocation for these molecules was lateral diffusion in the membrane, rather than fast axonal transport. This suggestion was recently confirmed in a striking manner by Codement et al. ${ }^{3}$, when they showed that these dyes can be used to label axonal projections in fixed tissue. Labelling with carbocyanine dyes has already allowed several exciting advances in developmental neurobiology. In this article we review the properties of carbocyanine dyes and point out some of their uses and advantages.

Neurobiology experienced a revolution when horseradish peroxidase (HRP) tracer methods were introduced in the early $1970 s^{4,5}$. HRP was first used for retrograde mapping of long-distance neuronal projections, but has proved to be very versatile. In many situations it can be used as an anterograde tracer, it is a superb intracellular marker, and it can also be localized at the electron microscopic level. However, HRP is not an ideal tracer in all situations. In particular, combining HRP with other labels can be difficult. This limitation led Kuypers and others to test a series of fluorescent molecules with different emission spectra as possible retrograde tracers. A number of such compounds are now widely used for this purpose $\mathrm{e}^{6,7}$.

$A$ second major limitation of HRP methods became apparent in the early 1980s. Physiological and developmental experiments on cultured neurons had always been limited by the inability to distinguish between the different types of neurons present in cultures made from CNS tissue. A number of investigators realized that one could inject HRP into the appropriate target region of the intact animal, wait for retrograde transport to occur before preparing cultures, and then later unequivocally identify neurons in culture that had projected to that target ${ }^{8-10}$. However, the reactions for visualizing HRP are normally performed on fixed tissue. This presented a problem, since fixed cells do not grow and have uninteresting electrical properties. The need to identify living neurons in culture motivated the search for fluorescent tracers that might be retrogradely transported in neurons in vivo and then retained during the dissociation process and in culture. The initial breakthrough was reported in 1980 by McPheeters and Okun who showed that $a$ Lucifer yellow conjugate of wheat germ agglutinin could be used to label motoneurons retrogradely, and was retained for a short time in culture ${ }^{11}$. This method was used by others ${ }^{12,13}$ to study the properties of developing motoneurons. However, with wheat germ conjugates, the fluorescence, even initially, was rather dim and restricted largely to small granules in the cell body with virtually no labelling of neuronal processes. In addition, the fluorescence diminished rapidly with time in culture, so labelled cells could be identified for only a few days. This problem could be overcome by running suspensions of spinal cord neurons through a fluorescence-activated-cell-sorter, and placing only the fluorescently labelled cells into culture. Since cell sorting produced pure populations of labelled cells it did not matter that the marker was no longer detectable after a few days. However, such an approach was not feasible for those investigators without ready access to a fluorescence-activatedcell-sorter and did not allow studies of interactions between different classes of neurons.

Our interests lie in understanding the cell-cell interactions that occur during axonal outgrowth and synaptogenesis. We wanted to take a cell culture approach, but we felt we first had to be absolutely certain about the identities of the different neurons present in each culture dish. We therefore began, in 1984, to survey fluorescent molecules in search of better labels. Our criteria for an appropriate tracer were that it should be retained in cells in culture for at least one week and that it should label processes as well as cell bodies.

We first tested a series of fluorescent compounds (e.g. True blue) that had previously been used as retrograde tracers in the mature CNS. Many of these were retrogradely transported from the periphery to the spinal cord of chick embryos, but all proved unsatisfactory in one way or another. We also tried rhodamine, which had been used very successfully by Bonhoeffer and Huf ${ }^{14}$ to label growing axons in culture. Although rhodamine did not persist in cells for as long as we required, we were struck by the manner in which it labelled processes and noted that it appeared to label the membrane, rather than the underlying cytoplasm. With this in mind, we therefore decided to survey fluorescent compounds that were known to label membranes. The carbocyanine dye dil $\left[1,1^{\prime}\right.$-dioctadecyl-3,3,3',3'-tetramethylindo-carbocyanine perchlorate; dil- $\left.\mathrm{C}_{18}-(3)\right]$ was among the first of those potential probes that we examined. The results with this dye were so striking that we did not extend our search much further. A paper reporting our initial experiments with carbocyanine dyes was published in $1986^{2}$. Here we summarize our results and describe the various ways we and others have used these dyes.
Marcia G. Honig is at the Department of Anatomy and Neurobiology, University of Tennessee Health Science Center, Memphis, TN38163, USA, and Richard l. Hume is at the Department of Biology, University of Michigan, Ann Arbor, MI 48109, USA. 


\section{Properties of carbocyanine dyes}

Dil is one of a large family of carbocyanine dyes that have been synthesized. Many different carbocyanine dyes are available commercially from Molecular Probes (Eugene, OR). The general structure of these compounds is shown in Fig. 1. The fluorochrome is a linked pair of conjugated rings that vary only in the $Y$ position. The common groups substituted at position $Y$ are isopropyl, oxygen, or sulfur, which give rise to the dil, diO and dis families of dyes. The linkage between the pair of rings consists of an odd number of carbons. We have used predominantly the three carbon-linked forms. It is a combination of the $Y$ constituent and the length of the linkage that determines the absorption and emission properties of the dyes. Attached to the fluorochrome are two long alkyl chains, which determine the affinity of the probes for membrane. Short-chain carbocyanine dyes partition between the aqueous and membrane phases in a membrane-potential-dependent manner, while the long-chain carbocyanine dyes are essentially insoluble in water and become inserted into cells with their alkyl chains embedded in the lipid bilayer.

We have used only two of these dyes very extensively. Dil is maximally excited in the green, and fluoresces bright red-orange when viewed through rhodamine filters, while diO [3,3'dioctadecyloxa-carbocyanine perchlorate; diO- $\mathrm{C}_{18}$ (3)] is excited in the blue, and fluoresces green when viewed through fluorescein filters. DiO fluorescence cannot be seen with rhodamine filters, but the excitation spectrum of dil is very broad and dil produces a goldish fluorescence when viewed with fluorescein optics. This fluorescence is not nearly as bright as the fluorescence viewed with rhodamine optics, but it offers the advantage that dil and diO can be viewed through the same filter set. Both of these dyes are intensely fluorescent, and this fluorescence fades much more slowly than either fluorescein or rhodamine fluorescence.

\section{Uses of carbocyanine dyes}

Labelling of plasma membranes. The long-chain carbocyanine dyes have been used extensively by cell biologists as probes of the fluidity of the plasma membrane (see Ref. 2 for a summary and references). Photobleaching studies indicate that these molecules move transversely in the membrane at a rate close to that calculated for a freely mobile molecule $\left(D=10^{-7}-10^{-8} \mathrm{~cm}^{2} / \mathrm{s}\right)$. Thus, most of the lipid bilayer is assumed to be fluid. In most experiments using carbocyanine dyes as probes of membrane fluidity, the dye was prepared as an ethanolic stock, and the stock was diluted in an aqueous buffer. Cells were then labelled by a brief incubation in the buffer. This method of application stains the entire plasma membrane of all cells. Similar methods were used by Schwartz and Agranoff $^{15}$ to stain neurons in culture with dil several years before we first used the dye. However, in these experiments the cells were viewed immediately after dye application, so nothing was learned about the properties of these dyes in living neurons or about how the dyes might affect the properties of living neurons. We expanded on this way of labelling cells by using virtually identical methods to label living neurons before they were placed into culture and maintaining them in culture for an extended length of time. We used this approach to label all the neurons in each of several peripheral ganglia (ciliary ganglia, sympathetic chain ganglia, dorsal root ganglia) with dil or diO.

For the first two or three days in culture, the neurons were brightly labelled in both their cell bodies and their processes (Fig. 2). Processes were often well-labelled out to the finest details of the filopodia on their growth cones. Since during dissociation neurons are stripped of their processes, this result indicated that dil (and $\mathrm{diO}$ ) could spread along the growing processes of neurons at a high rate. After a few days in culture much of the label appeared to be in cytoplasmic vesicles rather than in the plasma membrane, and the process labelling became much dimmer. However, some labelled cells could be identified by their granular cytoplasmic labelling for as long as three weeks in culture. The growth and electrophysiological properties of chick sympathetic and ciliary ganglion neurons labelled with either dil or diO gave no indication that the dyes were in any way toxic at the concentrations at which we introduced them into the membrane. However, although the brief fluorescent illumination necessary to identify cells did not seem to be damaging, subsequent time-lapse studies have indicated that prolonged fluorescent illumination at full intensity can cause damage. Damage can be minimized by attenuating the exciting light with neutral density filters, and viewing the emitted fluorescence with a low-light video camera and a digital image processor.

Retrograde labelling. At the same time as we were characterizing the properties of dil-and diO-labelled ganglion cells, we made the more significant discovery that dil could be used as a retrograde label which was retained during the dissociation process and could, therefore, be used to identify living cells in culture. We first demonstrated that retrograde labelling was specific. We injected dil into individual chick hindlimb muscles and found that the location of retrogradely labelled motoneurons within the lateral motor column corresponded to the position of the appropriate motoneuron pool as had previously been determined by HRP transport ${ }^{16}$. We next made spinal cord cultures from embryos in which we had retrogradely labelled motoneurons by injecting dil into major nerve trunks or retrogradely labelled sympathetic preganglionic neurons by injecting dil into the sympathetic chain ganglia. In each type of culture, the dye was retained by the retrogradely labelled neurons when they were placed into culture, and did not spread to other spinal cord cells. The characteristics of the labelling were virtually identical for retrogradely labelled cells and cells labelled during dissociation. Quite remarkably, the neurites and growth cones of retrogradely 
labelled cells were brightly fluorescent for the first one to two days in culture (Fig. 3), allowing studies not previously possible of growth cones of identified cell types ${ }^{17}$ (Fig. 4). Further, retrogradely labelled neurons could be identified for several weeks in culture by their granular cytoplasmic fluorescence (Fig. 5).

Anterograde labelling. In the course of these experiments, it almost immediately became apparent that dil also spread anterogradely in vivo. When we examined sections of chick embryos to assess the specificity of retrograde labelling following dil injections into nerves, we were struck by the axonal labelling we saw more distally in the limb (e.g. Fig. 6). Landmesser and Honig took advantage of this anterograde labelling to compare the outgrowth of sensory neurons and motoneurons into chick hindlimb ${ }^{18}$.

Several recent papers by others also illustrate the ability of anterograde labelling with carbocyanine dyes to resolve important issues ${ }^{19-23}$. A particularly striking example is the work of O'Leary and Terashima ${ }^{21}$ examining the growth of axons from layer 5 of the rat visual and motor cortex. Previous work had shown that the axons of these cortical neurons reach the base of some target regions (such as the pons) several days before they invade those targets. It had been suggested that the growth cones of these cells cease extending, and await a signal from the target to restart growth. One major conclusion from the new work is that the 'waiting period' after axons reach the level of the pons is not explained by growth cones pausing at the base of the pons. Rather, growth cones initially extend past the pons and grow to the more distant spinal cord. Several days later, collateral branches form, at the level of the pons, from axons already projecting to the spinal cord, and these new collaterals then invade the pons. Recent work in other systems, also using dil, similarly suggests that the formation of collaterals may be a more common mechanism underlying branching in vivo than is growth cone bifurcation 22 .

This type of experiment would not have been possible with most other neuronal tracers since they do not allow high-resolution labelling of individual axons at discrete points in time. Another exciting application of dil labelling is the ability to follow dynamic changes of individual growing axons in vivo. For example, Harris et al.22 have performed time-lapse studies of the growth of retinal axons in the tectum of Xenopus embryos (see also Ref. 23). A similar approach is currently being taken to look at the outgrowth of motoneuron axons in zebrafish (Eisen, J., pers. commun.).

Labelling in fixed tissue. Several observations led us to the conclusion that most of the spread of the dyes was by lateral diffusion in the plasma membrane. Among these were the location of the dye (mostly in the plasma membrane rather than in cytoplasınic vesicles) and the rate and bidirectionalitv cf 'transport'. We found that significant retro- measured for dil diffusion in other membranes. This idea has received striking confirmation by more recent work from Bonhoeffer's laboratory. Godement et al. ${ }^{3}$ showed that dil can work as an anterograde and a retrograde tracer in fixed tissue. In fact, in some situations there are advantages to performing tracing studies on fixed rather than living tissue. (1) Tracing studies can be performed in systems where access to target structures is otherwise difficult or impossible, for example in structures that lie deep within the brain and can only be approached in partly dissected or thick-slice preparations, in mammalian fetuses and in the human nervous system ${ }^{24}$. (2) The tissue can be kept as long as necessary for movement of the dye before viewing. (3) The dye is not internalized by labelled cells. The second and third points are particularly important because diffusion is a relatively slow process. In living tissue, dil produces significant labelling within about $6 \mathrm{~mm}$ of the injection site by one day ${ }^{2}$ and over a four-day survival period, labelling may extend up to $2-3 \mathrm{~cm}^{21}$. In living tissue, during long survival times, the membranous labelling is likely to be largely replaced by a granular cytoplasmic labelling, as the dye becomes internalized by endocytosis. As a consequence, labelled axons may become hard to visualize, possibly before they are labelled to their ends. In contrast, in fixed tissue, labelling appears to occur more slowly than in living tissue ${ }^{3}$, but fixed specimens can be kept for several weeks (or months, if necessary) after dye application to allow diffusion of the dye over a suitable distance. During this time, since endocytosis is not occurring, neurons and their processes acquire and retain a uniform membranous labelling.

Long-term labelling. A second class of experiments using dil and diO takes advantage of the long time that living cells remain labelled. We have used dil to identify sympathetic preganglionic neurons in cell culture over a two-week period. Our experiments have looked at synapse formation ${ }^{25}$ and at the regulation of expression of neurotransmitter receptors during development ${ }^{26}$. Although experiments using dil and diO as long-term labels have provided exciting new results, a particular concern is how long the labelling lasts in cultured neurons. We found with labelled sympathetic and ciliary ganglion neurons that most cells were still fluorescent at two weeks, but by three and a half weeks a substantial proportion of cells no longer had detectable fluorescence. In contrast, dil labelling has been reported to persist in vivo for up to nine months after application when it is used as a retrograde tracer in adult nervous system ${ }^{27}$. The gradual loss of labelling in cultured neurons may be a consequence of the extensive membrane addition and turnover that occurs in rapidly growing cells. An alternative to dil for long-term identification of retrogradely labelled neurons in culture might be the rhodamine-labelled latex microspheres introduced by Katz, Burkhalter and Dreyer ${ }^{28}$, though, to our knowledge no studies have compared the persis- 


\section{ARBOCYANINE DYES \\ Novel Markers for Labelling Neurons}

Marcia G. Honig, Department of Anatomy and Neurobiology, University of Tennessee Health Science Center, Memphis, TN 38163 , Richard I. Hume, Department of Biology, University of Michigan, Ann Arbor, MI 48109, USA.

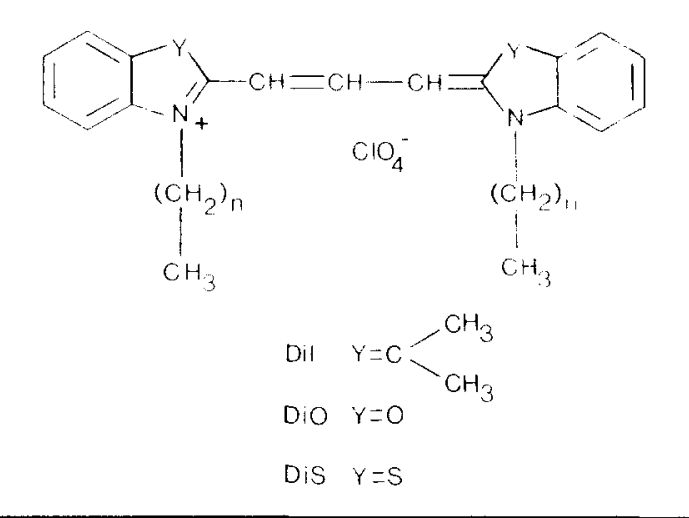

Fig.1. Chemical structure of carbocyanine dyes. The group at position $Y$ determines the type of dye (i.e. dil $\mathrm{diO}, \mathrm{diS})$. For the lipid-soluble dyes we use to label neurons $n=17$.

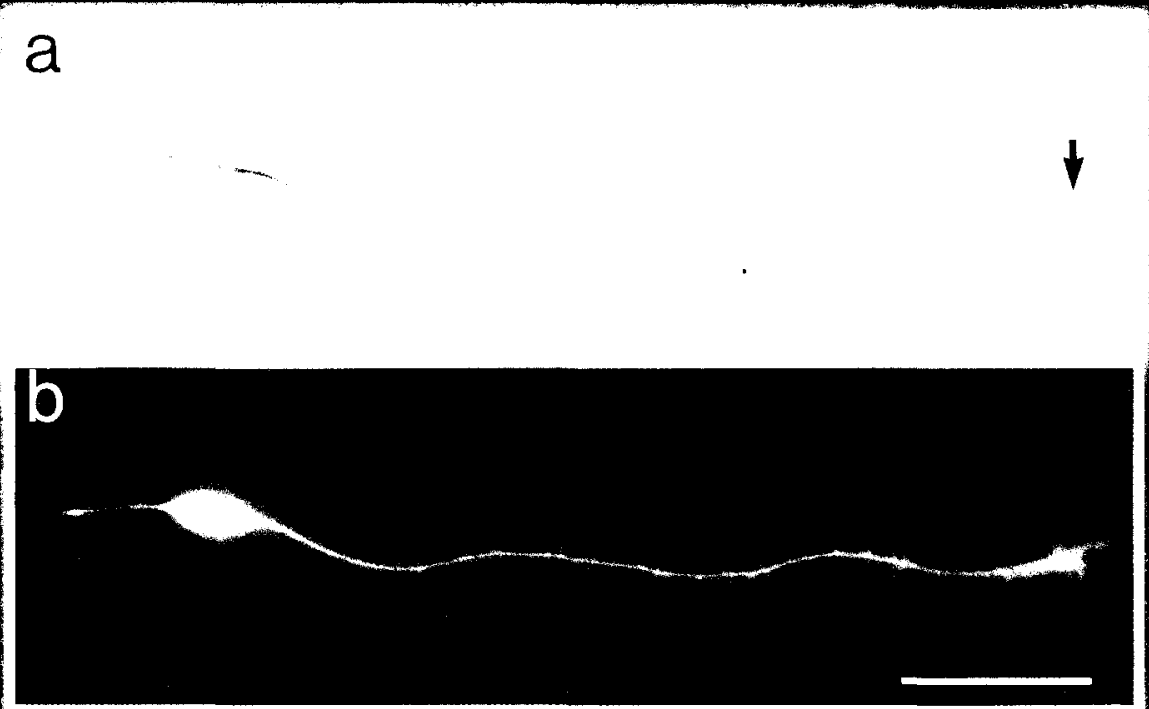

Phase-contrast $(\mathbf{A})$ and fluorescence $(\mathbf{B})$ views of a dorsal root ganglion neuron from a stage 29 chick embryo labelled by incubation in a dilute solution of diO during the dissociation procedure. Note that the processes this cell grew in culture are labelled, as is the growth cone (arrow) and even the fine filopodial extensions. The cell body appears yellow because the film becomes saturated during the long exposure needed to show growth cone labelling. This cell was in culture for 1 day Calibration bar $=50 \mu \mathrm{m}$.

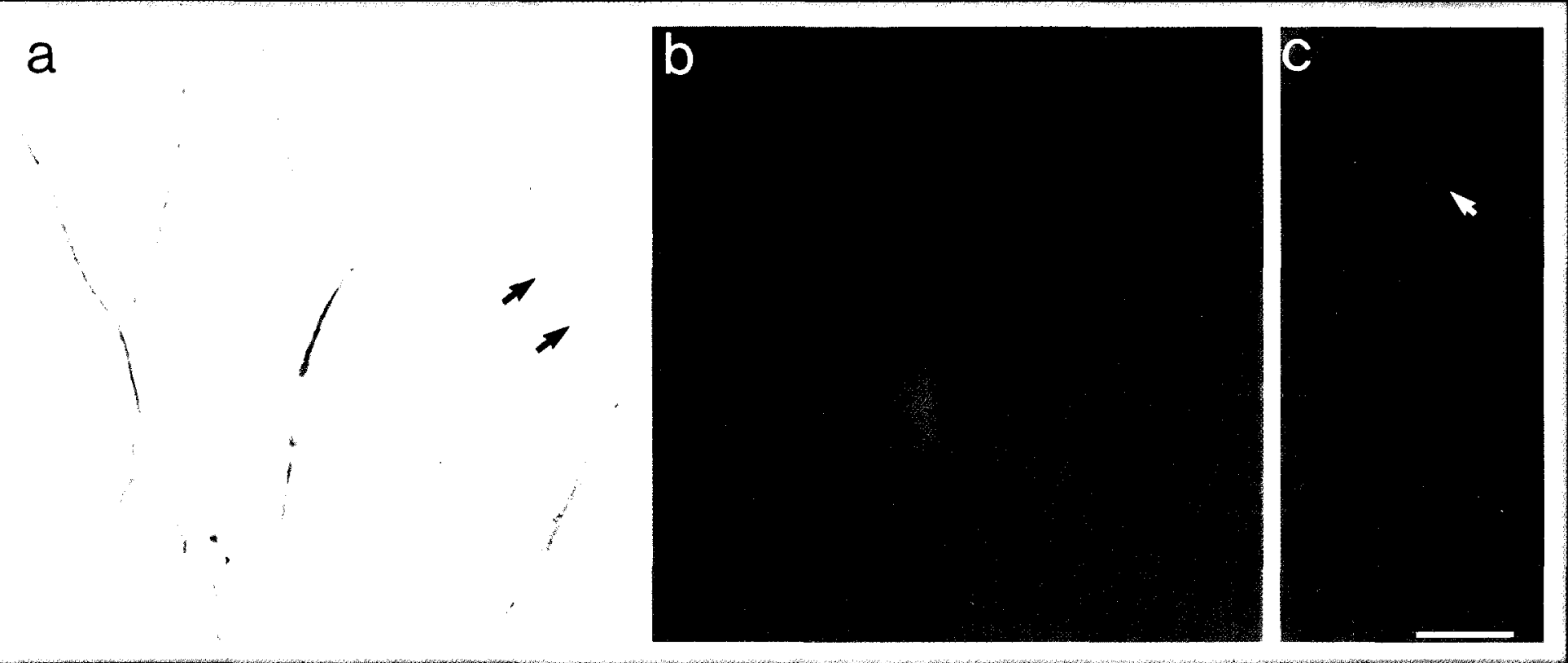

Phase-contrast (A) and fluorescence (B) views of a spinal cord culture from a stage 26 chick embrvo in which the motoneurons had been retrogradelv labelled bv iniec 


\section{a}

Fig. 4. Identification of different ts Phase-contrast (A,C) and double fluorescence (B,D) views of a spinal cord and dorsal root ganglion co-culture. The dorsal root ganglia were label (red). as described for Fig. 3. (A) and (B) show a sensory neuron growth cone [arrow in (A)] contacting a motoneuron neurite. (C) and (D) show a mot view. The spinal cord neurons had been in culture for 2 days, the dorsal root ganglion neurons for 1 day before these photographs were taken. Cali

Carbocyanine dyes (structure shown in Fig. 1) are a family of intensely fluorescent molecules, with a number of useful and unique applications as neuroni Two of the 18 carbon chain dyes, dil- $\mathrm{C}_{18}$-(3) or 'dil' for short, and $\mathrm{diO}-\mathrm{C}_{18}-(3)$ or 'diO', which fluoresce red and green, respectively, have been the mos chains make these molecules lipophilic, so that cells exposed to these dyes rapidly incorporate them into their plasma membranes. Once in the membran of native lipids. As a consequence, even when dye is applied to only a portion of a neuron's surface (such as the axon or cell body), the entire cell st labelled. Thus these dyes are very suitable for both retrograde and anterograde tracing (Honig and Hume, 1986).

Carbocyanine dye labelling techniques were initially developed to allow the identification of different types of neurons in dissociated cell cultures (Hor strategy was to retrogradely label a single population of neurons in vivo, prior to culturing. For example, motoneurons can be labelled by injection of when the spinal cord cells are cultured, the motoneurons can be distinguished from other types of spinal cord neurons also present in the cultures (Fi second population of neurons, for example those in peripheral ganglion, can be labelled by incubating the cells directly in dye during the dissociation pre is incorporated into the plasma membrane and during the first few days in culture, it brightly labels not only the cell body, but also the neurites that grou label one group of neurons selectively, and $\mathrm{diO}$ to label a second group of neurons selectively, one can readily distinguish between the two types of neurc in culture, as membrane is endocytosed, the dye becomes internalized but is still readily visualized for long times in culture (Fig. 5). The dyes do not ap in vivo during the dissociation process, or in culture, although a small amount of trans-cellular labelling has been detected under some conditions ( 1 Bonhoeffer, 1987). Furthermore, the dyes do not seem to interfere with the growth or electrophysiological properties of the neurons. Thus, with this the properties of individual identified neurons or their processes and to study interactions between different types of neurons in culture (Honig, 1987; Hu

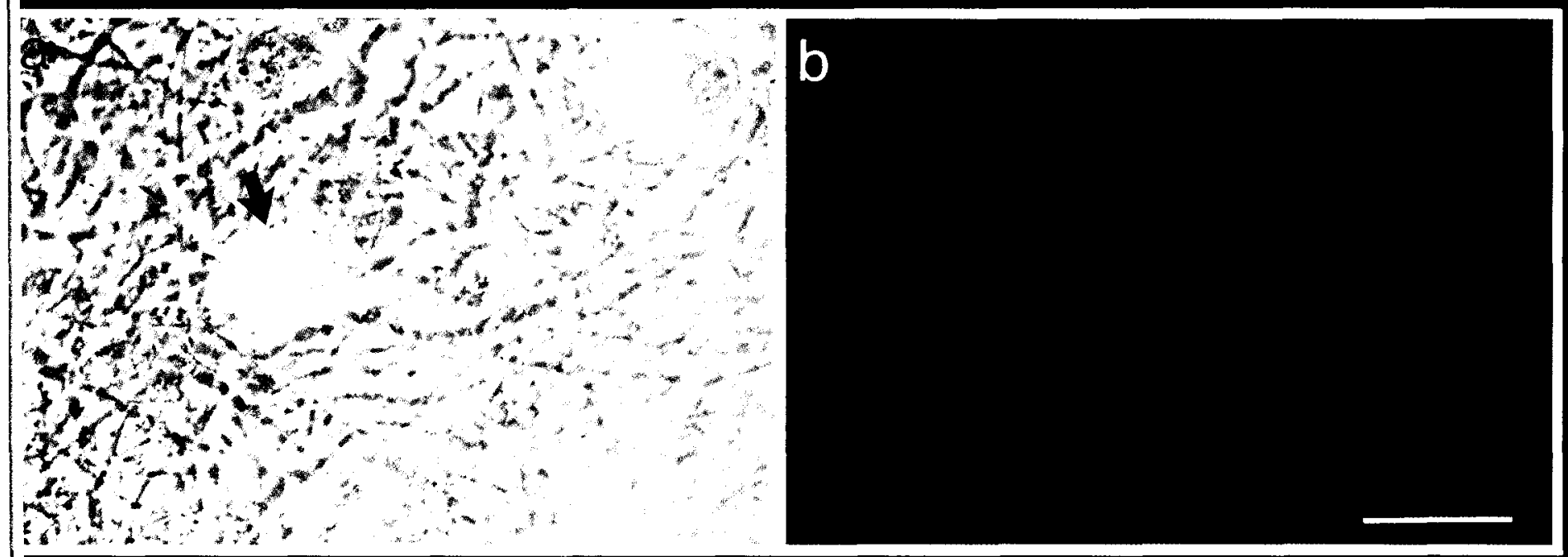




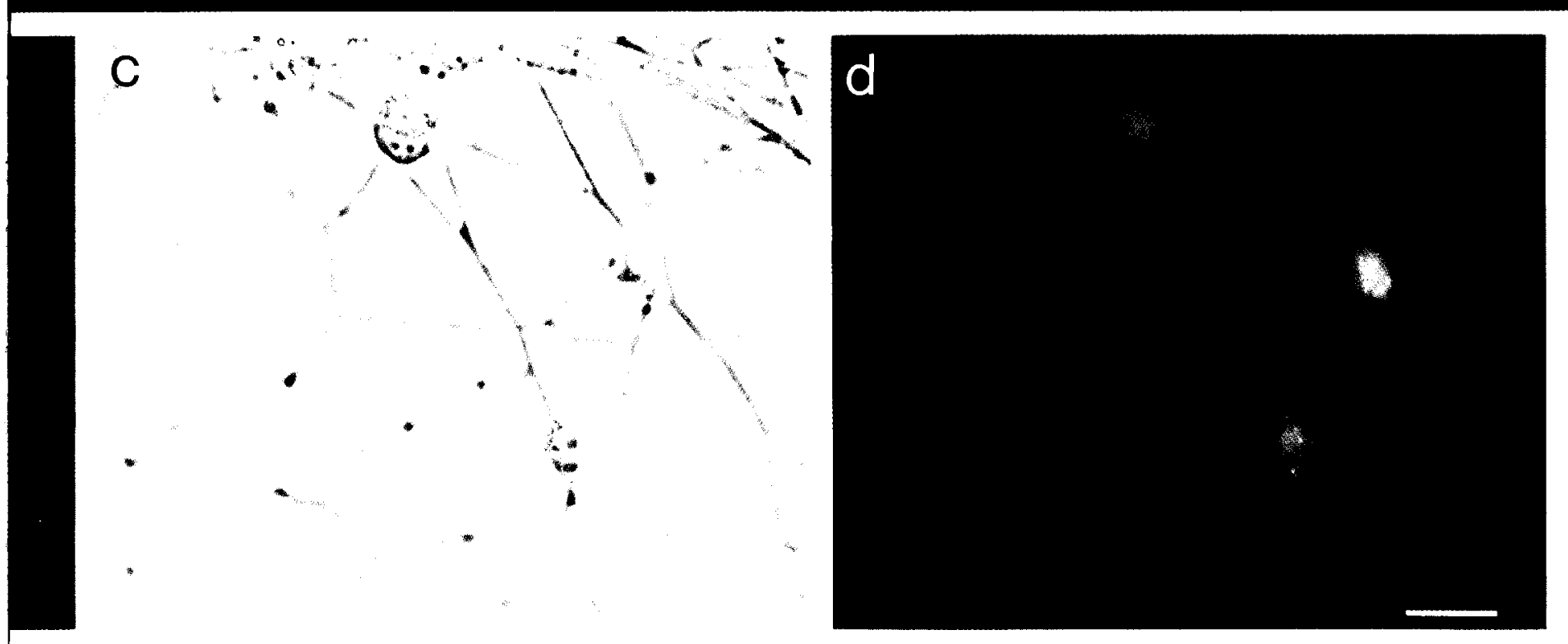

\section{pes of neurons and their processes in co-cultures.}

ed by dissociation in diO (green) as described for Fig.2: the spinal cord was from a second embryo in which the motoneurons had been retrogradely labelled unth dil oneuron. two sensory neurons and their labelled processes. The unlabelled processes originate from other spinal cord neurons whose cell bodies are out of the field of ration bar $=20 \mu \mathrm{m}$.

1 markers and for pathway tracing. frequently used. The long carbon 2 , the dyes diffuse with the mobility urface eventually becomes brightly

lig and Hume, 1986). The general iil into the spinal nerves, and later 3.3). Alternatively or in addition, a cess (Fig. 2). In both cases, the dye out of it (Figs 2,3). By using dil to ns in co-cultures (Fig.4). Over time pear to pass from cell to cell either jodement, Vanselow, Thanos and pproach, it is possible to examine me and Honig, 1987).

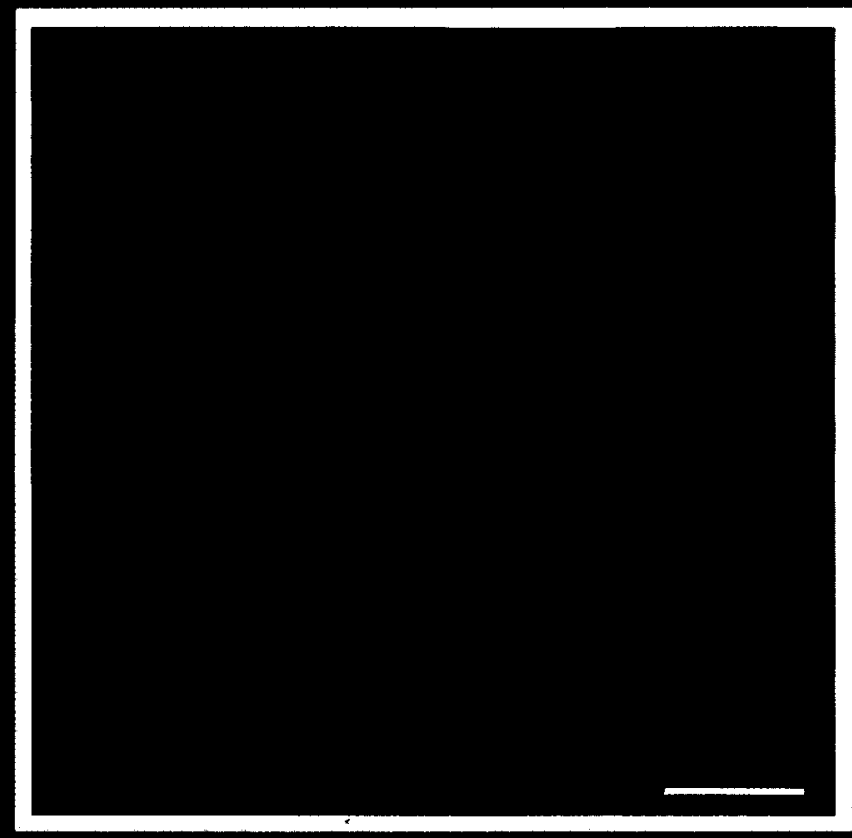

Fig. 6. Anterograde labelling. Fluorescence view of a vibratome sec tion from a stage 26 embryo showing motoneuron axon labelling in the sciatic nerve. Motoneuron axons were anterogradely labelled by injection of dil into the spinal cord. The embryo was fixed 16 hours after injection. Proximal is up. Labelled axons can be seen coursing distally and separating into two nerve branches. Individual axons are sometimes hard to resolve because many axons are labelled at varying planes of focus. Calibration bar $=100 \mu \mathrm{m}$.

\footnotetext{
bocyanine dyes have found their widest use in studies of axonal growth in the developing nervous system. Dil and diO are especially useful for anterograde tracing 6) since they allow high-resolution labelling of individual axons at discrete points in time. With this approach, several elegant studies have been performed showing $r$ axonal projections change during development (e.g. see O'Leary and Terashima, 1988). In addition, since labelling can be visualized in living tissue, it is possible ollow dynamic changes of individual growing axons and their growth cones in vivo (Harris, Holt and Bonhoeffer, 1987).

major advantage of carbocyanine dyes is that they can be used to label neurons in fixed tissue (Godement et al., 1987). Since they spread by diffusion rather than active transport, they are reliable tracers as long as the cell membranes remain intact. The use of fixed tissue allows experiments to be performed in situations re access to target structures would be difficult or impossible in living tissue, for example, in mammalian fetuses. A particularly exciting application of labelling d tissue is that it should be possible, by the use of post-mortem tissue, to map neuronal projections in the human brain (Burkhalter and Bernardo, 1989)
}

\section{cted References}

lalter. A. and Bernardo. K.L. (1988) Proc. Natl Acad. Sci. USA 86, 1071-1075

ment. P.. Vanselow. J. Thanos. S. and Bonhoeffer. F. (1987) Development 101, 697-713

5. W.A. Holt. C.E. and Bonhoeffer. F. (1987) Detelopment 101. 123-133

I. MG (1087) Neurosci Ahstr 13,1220

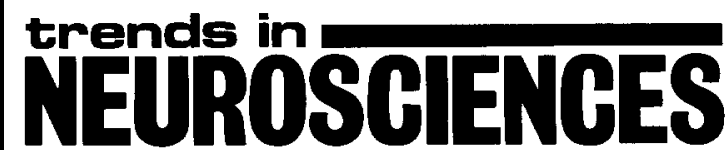



Fate mapping. Several investigators have taken advantage of the persistence of dil labelling by using it to follow a subpopulation of cells during development [chick somitic and somatopleural cells (Lance-Jones, C., pers. commun.); newt blastoma cells ${ }^{29}$ ]. For example, recent work from BronnerFraser's laboratory ${ }^{30}$ reports that dil can be used to study cell migration in developing mammals. Neural crest cells in mouse embryos were labelled with dil, and fluorescent cells were localized at a later stage in development. This method allows high-resolution mapping of cell fate that was previously possible in avian embryos (by the use of chick-quail chimeras) but not in mammals. Furthermore, it offers the advantage that the transplantation procedure and/ or differences between two species do not complicate the interpretation of the results. However, here too, there is a concern about how long the label lasts and, in particular, at what point it becomes so diluted by cell division that it can no longer be seen. For fate mapping over a period of more than a few weeks, an alternative approach might be to use a retroviral marker ${ }^{31}$. The major advantage of retroviruses is that DNA replicates during mitosis, so the label is not diluted when cells divide, and thus is essentially permanent. However, since it is simple to apply and view dil and it is possible to make repeated observations, if desired, it seems likely that dil will become widely used for many studies where cells are followed for intermediate lengths of time.

\section{Methods for dye application}

Dil and diO can be applied in a variety of ways, each of which may be best suited for different purposes. In our original studies, we dissolved dil in ethanol and diO in a mixture of ethanol and DMSO. These solutions were ejected from micropipettes for retrograde or anterograde labelling, or were diluted further in culture medium for direct labelling of cell suspensions or cell cultures. An alternative approach is to dissolve the dyes initially in dimethylformamide (DMF). This has the advantage that diO, in particular, is more soluble in DMF than it is in ethanol. In addition, it seems to be easier to limit the size of the injection site. In fact, by using DMF, finetipped microelectrodes and very short duration pulses of pressure, it is possible to label just a single cell in an embryo of an organism with a simple nervous system such as a zebrafish (Eisen, J., pers. commun.; Kuwada, J., pers. commun.).

In other experiments, one might wish to label large numbers of neurons or axons. To do this, it may be easier to apply crystals of the dyes, rather ihan injecting a solution, especially in fixed tissue where dye spread from an injection site would be minimal ${ }^{3}$. Other ways to apply dil and $\mathrm{diO}$ are discussed by Godement et al. ${ }^{3}$ and by Vidal-Sanz et al. ${ }^{27}$.

\section{Limitations on the use of carbocyanine dyes}

A critical issue in our initial paper was whether

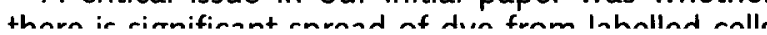

number of labelled motoneurons counted shortly after plating was never greater than the number of neurons expected to be retrogradely labelled. (2) We did not observe any double-labelled cells in cultures of ciliary ganglion neurons in which all cells had initially been labelled either red with dil or green with diO. (3) When individually labelled preganglionic neurons were followed over time in culture their neighbors did not become labelled. It is important to note that these controls do not mean that carbocyanine dyes could not spread from cell to cell under other circumstances. In fact, we occasionally saw lightly labelled ependymal cells in spinal cord cultures containing retrogradely labelled sympathetic preganglionic neurons ${ }^{2}$, and Godement et al. sometimes observed labelled cells in very close apposition to very brightly labelled axons ${ }^{3}$. However, in both cases the secondary 'transcellular' labelling could be distinguished from the primary labelling. None the less, it is crucial that the possibility of dye transfer be evaluated in each new situation in which these dyes are used.

Experiments using carbocyanine dyes are also complicated by the failure to find a way to fix the dyes to the tissue. As long as the membranes are intact (in living cells or fixed whole mounts) this is not a problem, but there are problems with studying sectioned material. The dyes seem to diffuse when membrane integrity is disrupted during sectioning. This problem is not very serious for anterograde tracing, since axons are so thin that a section of moderate thickness is often equivalent to a whole mount. However, for retrogradely labelled cell bodies, if many labelled cell bodies are sectioned, there is a considerable source of mobile dye which may diffuse away from the true site of labelling.

One way to, in part, circumvent this fixation problem would be to use a photo-oxidation method, as has previously been described for other fluorescent markers ${ }^{32,33}$. According to this approach, excitation of the fluorescent dye in the presence of diaminobenzidine results in the deposition of an insoluble diaminobenzidine reaction product. Success in carrying out this procedure with dil has recently been achieved by S. McConnell, A. Ghosh and $C$. Shatz (pers. commun.), and the expectation is that this advance will allow dil labelling to be visualized at the EM level.

\section{Concluding remarks}

Long-chain carbocyanine dyes are intensely fluorescent, and fade relatively slowly when exposed to exciting light. They are rapidly incorporated into membranes, and diffuse freely in the plane of the membrane. We found that the carbocyanine dyes dil and diO label neurons retrogradely and anterogradely, and can therefore be used for tracing neuronal projections. The anterograde labelling allows one to visualize growing axons with high resolution and several investigators now use this approach for dynamic studies of 
identify and study the properties of those neurons in culture. In addition, we label a second population of neurons by a brief incubation in diO, enabling us to examine interactions between two identified types of neurons in culture. In many cases, dil and diO let the investigator perform experiments that would not be possible with other neuronal tracers.

\section{Selected references}

1 Honig, M. G. and Hume, R. I. (1985) Neurosci. Abstr. 11, 98

2 Honig, M. G. and Hume, R. I. (1986) J. Cell Biol. 103, 171-187

3 Godement, P., Vanselow, J., Thanos, S. and Bonhoeffer, F. (1987) Development 101, 697-713

4 Kristensson, K. and Olson, Y. (1971) Brain Res. 29, 363-365

5 LaVail, J. H. and LaVail, M. M. (1972) Science 176 1416-1417

6 Skirboll, L. et al. (1984) Brain Res. 8, 99-127

7 Schmued, L. C. and Fallon, J. H. (1986) Brain Res. 377. 147-154

8 Bennett, M. R., Lai, K. and Nurcombe, V. (1980) Brain Res. $190,537-542$

9 Tosney, K. W. and Landmesser, L. T. (1981) Neurosci. Abstr. 7,550

10 Tanaka, H. and Obata, K. (1983) Dev. Brain Res. 9, 390-395

11 McPheeters, M. and Okun, L. M. (1980) Neurosci. Abstr. 6, 733

12 Calof, A. L. and Reichardt, L. F. (1984) Dev. Biol. 106, 194-210

13 O'Brien, R. J. and Fischbach, G. D. (1986) J. Neurosci. 6 3265-3274
14 Bonhoeffer, F. and Huf, J. (1980) Nature 288, 162-164

15 Schwartz, M. and Agranoff, B. W. (1981) Brain Res. 206 , 331-343

16 Landmesser, L. (1978) J. Physiol. (London) 284, 371-389

17 Honig, M. G. (1987) Neurosci. Abstr. 13, 1220

18 Landmesser, L. and Honig, M. G. (1986) Dev. Biol. 118, 511-531

19 Thanos, S. and Bonhoeffer, F. (1987) J. Comp. Neurol. 261 , 155-164

20 Stuermer, C. A. O. (1988) J. Neurosci. 8, 4513-4530

21 O'Leary, D. D. M. and Terashima, T. (1988) Neuron 1, 901-910

22 Harris, W. A., Holt, C. E. and Bonhoeffer, F. (1987) Development 101, 123-133

23 O'Rourke, N. A., Fox, B. E. S. and Fraser, S. E. (1987) Neurosci. Abstr. 13, 368

24 Burkhalter, A. and Bernado, K. L. (1989) Proc. Natl Acad. Sci. USA 86, 1071-1075

25 Hume, R. I. and Honig, M. G. (1987) Neurosci. Abstr. 13 1425

26 Clendening, B. and Hume, R. I. (1987) Neurosci. Abstr. 13,1227

27 Vidal-Sanz, M., Villegas-Perez, M. P., Bray, G. M. and Aguayo, A. J. (1988) Exp. Neurol. 102, 92-101

28 Katz, L. C., Burkhalter, A. and Dreyer, W. J. (1984) Nature 310, 498-500

29 Ferretti, P. and Brockes, J. P. (1988) J. Exp. Zool. 247, 77-91

30 Serbedzija, G., Fraser, S. and Bronner-Fraser, M. (1988) Neurosci. Abstr. 14, 427

31 Sanes, J. R. (1989) Trends Neurosci. 12, 21-28

32 Maranto, A. R. (1982) Science 217, 953-955

33 Sandell, J. H. and Masland, R. H. (1988) J. Histochem. Cytochem. 36, 555-559
Acknowledgements Ourinitial work using carbocyanine dyes was supported by NIH grant NS21043 to RH. Our current work using these dyes is supported by NIH grant NS21043 to RH and NS26386 to $\mathrm{MH}$

\section{Special Issue}

The next special issue of Trends in Neurosciences will be a follow-up to last year's popular and successful issue on calcium. It will contain a selection of articles focusing on the involvement of calcium in intracellular processes, including second-messenger systems, gene expression and cytoskeletal modelling. This special issue has been planned with the assistance of Mary Kennedy. The following articles will be included:

Calcium control of ion channels, by Alain Marty

The EF-hand family of calcium-modulated proteins, by Robert Kretsinger

The mechanism of action of protein kinase $C$, by Kuo-ping Huang

The calpains, by Sandro Pontremoli

The impact of postsynaptic calcium on synaptic transmission - its role in longterm potentiation, by Roger Nicoll

Calcium-sensitive and calcium-insensitive exocytosis, by Derek Knight

Stimulus-transcription coupling in neurons: role of cellular immediate-early genes, by James Morgan and Tom Curran

The mechanism and regulation of fast axonal transport, by Mike Sheetz and Trina Schroer

Calcium and polyphosphoinositide control of cytoskeletal dynamics, by Paul Forscher

If you are planning to present a course covering this area in the future you might be interested in buying extra back issues. Bulk orders (in multiples of ten) can be obtained at discount prices - for further details contact the editor.

If you are not a subscriber, why not take out a subscription today to ensure a copy? 